\title{
Research on Application of Core Strength Training in Sports Training
}

\author{
Li Shengfu, Department of Physical Education, Wuhan Institute of Technology, Wuhan, Hubei, 430205 \\ Wang Hanxi, Department of Physical Education, Wuhan Institute of Technology, Wuhan, Hubei, 430205
}

\begin{abstract}
Core strength is a new idea of strength training derived from the rehabilitation field in 1990's. At present, the core strength has been widely adopted in high level sports in our National Taekwondo team, canoeing, tennis team and basketball team. It has good effects to improve the motion performance. Core strength exists in all sports.Core muscle body plays a role in stability and support of posture in the movement skill and special technology action. This paper discusses the concept and function of core strength and its training methods to improve the understanding of core strength, and fully understand the importance and occurrence mechanism of core strength in sports.
\end{abstract}

KEYWORDS: Core strength; Sports training

\section{Introduction}

At present, the core strength training theory has been accepted by many Chinese scholars, coaches, athletes. NationalCanoeing Team, Taekwondo Team, Tennis team and Ice Team have started to core strength training.Core strength training from a biomechanical perspective will be under the condition of moving human body as a "chain". The body core area is the central links of the kinematic chain, providing a stable fulcrum for the transfer of the upper and lower extremity strength to improve the stability of sport technique.Core strength and core stability exercises can improve the strength of connection between different parts of the body to improve muscle work efficiency. Although the theories of core strength training is still controversial, core strength training has been used by more and more projects.

\section{Concept of Core Strength}

Core strength usually refers to the core of the torso, including the spine and pelvis and its surrounding muscles. The core we mean that the body below the ribs and pelvis area, it contains the muscles have abdominal muscles and back muscles, transverse pickled muscle and pelvic floor muscle, staggered pelvic and lower extremity muscles. Lumbar physiological mechanism of the core muscles of the pelvic hip includes 29 muscles are located in the core position of the human body. These muscles in the human body motion play roles in stabilizing balance, transmitting power and reducing the force effects.

The concept of core power originated from the core stability based on the stability of the spine. In 1985, Panjabi first proposed the concept of spinal stability. He believed that the stability of the spine includes muscle and neural control unit three system passive, active spinal vertebrae. Core strength is the human core body muscles, in order to stabilize the core parts of the body center of gravity control, transmission, movement of upper and lower extremity strength for the main aim of the power capacity, which is mainly influenced by many factors. The core muscles are affected by innervated ability support ability and the matching of 
breath and movements.

Core strength exists in all of sports items. At present, all sports action is a movement chain to center muscle group which plays a role in stability and support in motion posture, motor skills and special technology action.Any athletic project technical movements are not relying on a single muscle group can be completed.It must mobilize many muscles coordination work. For the stability of the center of gravity, a point, conduction power functions in the process of core muscles. It is also the main part of the overall force which plays a pivotal role on the upper and lower limb collaborative work.

\section{Effects of Core Strength in Sports Training}

\section{A: Raise Stability of Technical Action}

From the angles of biomechanics of the human body in motion,core strength training will be regarded as a chain.Each part of the body is a link on the chain, the movement technology to complete, depending on the momentum transfer in the chain to the core strength to play a pivot role in transfer process dynamic chain implementation, coordination of upper and lower limbs sports, improve the power transfer, to improve the technical stability. We use some of its non-steady state strength exercises and balance exercises in core strength to increase the training methods of the suspension training non steady state. Traditional strength training is in steady state.In the process of movement and broke the stable state of the body, which makes the strength to play a better role. Core strength training has obvious effect on core muscle strength improved. More emphasis on the control ability of nerve is put on the muscle to improve movement stability.

\section{B: Improve Muscle Work Efficiency}

Research found that the force at the core of a good sprinter during the flight period quantity is less which can make the muscles relaxes during the flight time, reduce unnecessary energy consumption while relaxing the muscles for the next contraction ready shot for shot motion. Each segment by the trunk fixed support point each link of bone around a fixed point, fixed point of bone for relative movement, need core stability participation, core stability will be lower extremity and trunk muscle forces of efficient delivery to the upper limbs, body force is applied to the instrument set. Standing triple jump in three, 100 and 800 meters run are lower limb as the last point of application of force of upper limb movement, also need the participation, and control the core muscles to state stability of the core area, is the upper and lower extremity strength transfer key in the process of running, the body center of gravity in along with the movement and change, center of gravity and large amplitude change will consume too much energy. Core strength is strong enough to control the center of the stability and reduce the energy consumption.

\section{C: Prevent Sports Injury}

Core strength and core stability are initially proposed to come from the field of rehabilitation.It itself is a kind of treatment of disease and injury means. Steady state contributes to the improvement of spinal strength and the core to improve the stability, avoid such as the occurrence of standing triple jump in three unbalanced lateral lumbar injury situation landing. At the same time, through the coordination role of the core area, it can reduce and buffer extremity and joint load by the establishment of a stable fulcrum for the upper and lower limbs strength to prevent the occurrence of sports injury. 


\section{Effects of Core Strength in Sports Training}

In recent years, core strength is a fresh thing in athletics sports. The core strength training is used in various sports teams. The core strength training has become a hot spot of physical training. It is very effective to develop the core muscle strength and body central balance stability and is also an effective means to improve the motion ability and injury prevention.

\section{A: Raise Competitive Ability of Sprint Athletes}

In an article of Research on Improving Women Sprinters Competitive Ability using Core Strength Training, the results of 24 weeks of core strength training of 6 female athletes from the Shandong Province Trackthe special form, the special function and quality have varying degrees of improve, special technology action is also different degree to be perfect, the performance of special high also has significant. Through core strength training, the athletes in the movement of the smooth movement ability was improved, the core parts involved in the control of trunk stable muscle groups and small muscles through core strength training.After the core strength training, the torso stability has been improved.

\section{B: Reduce Sports Injury of Rowing Athletes}

In an article of Core Strength Training Research in Youth Rowing Athletes, the importance of the core strength training of juvenile rowing athletes, and combining with the practical training introduces several core strength of conventional training methods, proposed reduces the proportion of anaerobic strength training, there was an increase in the low strength of the core strength training oxygen intensity training; reduce land strength training volume, increase in water balance and stability of various forms of special strength training exercises at night increase the core strength training, practice has proved, this will not only help to improve the force at the core, but also can effectively reduce the incidence of sports injury, contribute to the existing sports injury rehabilitation.

C: Enhance Stability of Landing in Freestyle Skiing Aerials Athletes

In an article of Freestyle Skiing Aerials Athletes Core Strength Training Research, through the study of the diagnosis and the core strength training of Chinese excellent Freestyle Skiing Aerials athletes, it has the important meaning to realize the core strength training on learning the 11 Freestyle Skiing Aerials project athletes movements in the air and ground, is to be able to stabilize the spine, pelvis mainly, to maintain the correct body posture, effectively complete the technical movement; improve the athletes in the air when the body control and balance; enhance the stability in the air when the body balance ability and landing.

In short, core strength refers to the body to produce displacement or rotation of the ability, the core area of stability of power refers to the body against displacement or rotation ability, core area stability control system, power is the core area of the core area of passive and active system stability muscles and respiratory regulation system matched with the functional performance, especially the stability the core area of the single joint, muscle, muscle fiber small short, such as the spine, waist, between iliac between small muscle strength.The function is to make the core area is more stable.

\section{Conclusions}

Core strength training has been the coaches and athletes' attention through the core strength training can effectively improve the athletes' function level, obtains the competitive sport 
level better. Core strength training helps keep your body movements and the correct sequence of skeletal lever, but also improve the balance ability, enhance the stability of movement. Core strength training is divided into static and dynamic strength training, strength training two.During the practice of training, the two methods should be combined closely. No matter the athlete is high level or low level,core strength training, like other strength training, should be gradualto combine the special technical training.

\section{REFERENCE:}

[1]Meng Xianfeng.Research on Improving Women Sprinters Competitive Ability using Core Strength Training,[J],Journal of Shandong Institute of Physical Education and Sports,2009,04

[21 Xue Bing.Core Strength Training Research in Youth Rowing Athletes[J],Journal of Hubei Sports Science, 2009,03

[3]Zhao Jia, Wang Weixing.Freestyle Skiing Aerials Athletes Core Strength Training Research [J],Journal of Shandong Institute of Physical Education and Sports,2009,05 